Mots. Les langages du politique

$80 \mid 2006$

La politique mise au net

\title{
Analyse sémiolinguistique des pages d'accueil des sites politiques suisses sur Internet
}

Marc Bonhomme et Pia Stalder

\section{CpenEdition}

Journals

Édition électronique

URL : https://journals.openedition.org/mots/484

DOI : $10.4000 /$ mots. 484

ISSN : 1960-6001

Éditeur

ENS Éditions

Édition imprimée

Date de publication : 1 mars 2006

Pagination : 11-23

ISBN : 2-84788-095-X

ISSN : 0243-6450

Référence électronique

Marc Bonhomme et Pia Stalder, "Analyse sémiolinguistique des pages d'accueil des sites politiques suisses sur Internet », Mots. Les langages du politique [En ligne], 80 | 2006, mis en ligne le 01 mars 2008, consulté le 23 avril 2022. URL : http://journals.openedition.org/mots/484 ; DOI : https://doi.org/ $10.4000 /$ mots. 484 


\section{Analyse sémiolinguistique des pages d'accueil des sites politiques suisses sur Internet}

«Vaisseau amiral du site Internet ${ }^{1}$, les pages d'accueil constituent à elles seules un microgenre qui justifie leur examen spécifique. Non seulement elles forment une hyperstructure complexe reflétant l'organisation tant globale que détaillée du site, mais elles sont une carte de visite souvent décisive pour le bon succès de son exploration. D’où le soin extrême que prennent les concepteurs dans leur élaboration formelle et rédactionnelle. Ces observations se vérifient avec les sites des partis politiques qui trouvent dans le média Internet un véhicule particulièrement adapté pour s’identifier, faire connaitre leurs positions et solliciter l'intérêt, voire l'assentiment de leurs visiteurs.

Cet article se propose de mettre en évidence les principales caractéristiques des pages d'accueil qui alimentent la communication informatique des partis politiques suisses. Notre analyse se fonde sur l'examen, le 13 janvier 2005, des pages des seize partis représentés au parlement helvétique, lesquels couvrent tout le spectre idéologique allant de la droite à la gauche ${ }^{2}$. À travers une étude comparative et fonctionnelle, nous verrons comment les partis concernés exploitent le support informatique de ces pages d'accueil selon une stratégie communicative fondée sur l'efficacité à court terme, que ce soit au niveau interactif ou

1. K. Meier, 2002, Internet-Journalismus, Constance, UVK Verlag, p. 90.

2. Voici la liste de ces partis, avec entre parenthèses, pour chacun, le sigle officiel et l'adresse Internet (à faire précéder de http://www.) :

- Droite : Démocrates suisses (DS : schweizer-demokraten.ch) ; Lega dei Ticinesi (Lega : legaticinesi.ch) ; Union démocratique du Centre (UDC: svp.ch) ; Union démocratique fédérale (UDF : udf.ch).

- Centre : Parti chrétien social (PCS: csp-pcs.ch) ; Parti démocrate-chrétien (PDC: cvp.ch) ; Parti évangélique suisse (PES : evppev.ch) ; Parti libéral suisse (PLS : liberal.ch) ; Parti radical démocratique (PRD : prd.ch).

- Gauche: Alliance socialiste (AS : solidarites.ch) ; Grünes Bündnis (GB : gb-aves.ch) ; Grünliberale Zürich (GLP: gruen-liberale.ch); Parti écologique suisse (Verts : gruene.ch); Parti socialiste (PS: sp-ps.ch) ; Parti suisse du Travail (PST : pda.ch) ; Sozialistisch Grüne Alternative Zug (SGA : sga-zug.ch).

Université de Berne, Section de linguistique française, marc.bonhomme@rom.unibe.ch,pia.stalder@rom.unibe.ch 
informatif. Une telle stratégie nous conduira à nous demander si ces pages d'accueil contribuent à la « démocratie électronique »³ ${ }^{3}$ qu'Internet est censé favoriser.

\section{Une forte sollicitation de l'internaute}

Globalement, les partis politiques suisses construisent leurs pages d'accueil en suivant le principe ergonomique qui régit ce microgenre: permettre à l'internaute une exploration maximale du site en un minimum de temps, de façon à réduire les risques de fourvoiement ou de «zapping » de sa part. À cet effet, les pages d'accueil examinées mettent en place un important dispositif de navigation, ce terme qualifiant les facultés de liaisons et d'échanges entre le système informatique et l'utilisateur, l'écran de l'ordinateur fonctionnant comme interface. De plus, sur le plan énonciatif, ce dispositif de navigation s'accompagne de toute une mise en scène dialogique, à travers laquelle les concepteurs visent à impliquer sur le champ l’internaute dans sa visite du site.

\section{Navigation}

Les sites des partis politiques suisses offrent deux types de pages d'accueil. Privilégiant le syncrétisme fonctionnel, la plupart d'entre elles (13 sur 16) combinent page d'entrée et page d'accueil en un seul ensemble, celui-ci amalgamant des éléments disparates plus ou moins mis en valeur selon les partis: marquages identitaires du site, formules de contact, instruments de connexion... Dans une démarche davantage procédurale, trois partis de gauche (PST, Verts, GB) choisissent une conception dédoublée de l'accueil, avec d'abord une page d'entrée comportant l'identité et la dénomination du parti. Ce n'est qu'après un clic sur le logo de la page d'entrée que l'internaute accède à la page d'accueil proprement dite. Mais dans les deux cas, les pages d'accueil se présentent comme des unités de lecture et des unités de sélection, en ce qu'elles orientent la circulation de l'internaute sur les niveaux inférieurs du site grâce à leurs barres de navigation, que l'on peut comparer aux sommaires de la presse écrite ${ }^{4}$. Sept pages analysées focalisent leurs ressources interactives sur une barre de navigation qui associe des rubriques hétéroclites (par exemple « Portrait », "Sections » ou « Politique de l'emploi » dans le site des Grünliberale). Cinq partis ont deux barres de navigation, organisées selon plusieurs modalités. Ainsi le Parti écologique dispose, à gauche de sa page, une première barre ouvrant sur quel-

3. N. Ethuin et R. Lefebvre, 2003, "Les balbutiements de la cyberdémocratie électorale », V. Serfaty éd., L'Internet en politique, Strasbourg, Presses universitaires de Strasbourg, p. 158.

4. Voir G. Otman, 1998, Les mots de la cyberculture, Paris, Belin, p. 284. 
ques rubriques de premier plan ("Votations fédérales », « Le bulletin vert»...). Mais le haut de sa page contient également une seconde barre d'arrière-plan, celle-ci donnant accès à des rubriques généralistes («Politique », «Liens »...). Trois pages de grands partis (PRD, PDC et PS) ont un système de navigation plus sophistiqué avec trois barres disponibles. Celles du Parti démocrate-chrétien se différencient notamment par les codes utilisés: listing verbal de rubriques ("Communiqués», "Agenda »...) sur la barre de gauche, éléments iconiques (comme une microcarte postale virtuelle) sur celle de droite, pictogrammes sur celle du haut. Entre autres, si l'on pointe sur le pictogramme figurant un drapeau stylisé et symbolisant la vie du parti, les mots clés relatifs à cette rubrique se mettent à défiler dans le bandeau entourant ce pictogramme.

Plus spécifiquement, la navigation avec le corps du site se fait au moyen des variantes libres que sont les éléments stratégiques de la page d'accueil, ce qui accroit le potentiel interactif de cette dernière. Les logos et les emblèmes des partis ont facilement une fonction « home» (GB, PES...). En particulier, la pauvreté technique de la page des Démocrates suisses est compensée par un élément saillant: au-dessus de la barre de navigation, non seulement on voit un cœur rouge qui bat à un rythme régulier, mais ce cœur fonctionne ultérieurement comme « retour à la page d'accueil », l'internaute nouant alors un contact étroit avec la figuration de cet organe hautement connoté. Tout autant stratégiques pour la portée communicative du site, les titres (GLP, SGA...) et les images (PRD, PS...) ont occasionnellement un rôle d'hyperlien. La page de la Lega dei Ticinesi (reproduite en Annexe 1) est symptomatique à cet égard: quand on clique sur l'icone animé représentant un panneau routier "Zoll», on accède immédiatement à la prise de position du parti à propos des accords douaniers de Schengen; un pointage sur le portrait de Giuliano Bignasca, le président de la Lega, suffit pour faire apparaitre une page blanche «mail» conviant l'internaute à correspondre avec celui-ci.

Si l'interactivité technique des pages d'accueil observées satisfait en gros le principe ergonomique énoncé supra, certaines d'entre elles ne sont pas exemptes de problèmes de navigation. Ces problèmes tiennent à des redondances manifestes: sur la page du Parti suisse du Travail, on peut cliquer indifféremment sur les titres du rédactionnel ou sur la fin du «lead» pour aboutir à la même rubrique dans le corps du site. Inversement, d'autres pages d'accueil pèchent par leur déficit en navigabilité. Par exemple, celle de la Sozialistisch Grüne Alternative correspond à l'intitulé «Aktuell » sans que cela soit signalé explicitement, si bien que l'internaute a des difficultés pour la retrouver lorsqu'il navigue sur le site.

Dans un pays plurilingue comme la Suisse, le choix des langues constitue un critère important pour le segment de population visé par le site, mais aussi pour la navigabilité des pages d'accueil. Il n'y a évidemment pas de sélection linguistique dans les sites monolingues des petits partis qui ciblent avant tout 
un public régionaliste ${ }^{5}$, en allemand (GB, GLP, SGA), en français (AS, PST) ou en italien (Lega); ni sur les pages qui mélangent des informations en allemand et en français (PCS). La sélection linguistique est limitée pour les sites majoritairement monolingues ayant une courte rubrique en d'autres langues. Ainsi, si la page d'accueil des Démocrates suisses est en allemand, ce parti met à disposition des listes de récolte de signatures en allemand, en français et en italien. Par contre, la sélection linguistique s'avère capitale pour les pages d'accueil des grands partis nationaux, qui offrent très clairement un choix entre deux langues (français et allemand pour le PRD et l'UDF) ou entre trois langues (deutsch, français, italiano pour le PDC). La sélection linguistique est maximale sur la page du Parti évangélique, formation religieuse à ouverture œcuménique, dont le portail est accessible en cinq langues: deutsch, english, français, italiano, rumantsch. Signalons que c'est le seul parti qui porte un petit égard à la quatrième langue suisse, très minoritaire, qu'est le romanche.

\section{De la navigation au dialogisme}

Au niveau discursif, la sollicitation de l'internaute prend la forme du dialogisme interlocutif ${ }^{6}$, lequel définit les relations des énoncés avec la compréhensionréponse de leurs destinataires réels ou virtuels, que l'on anticipe. Ancrant par avance la réception dans le discours, un tel dialogisme vise à compenser la dimension fugace et aléatoire de la communication politique par Internet. De plus, il est fragile, dans la mesure où il repose essentiellement sur des actes initiatifs dont la satisfaction rétroactive n'est nullement garantie. Sur la plupart des pages d'accueil des partis politiques suisses, ce dialogisme interlocutif s'exerce dans deux directions. D’un côté, il fournit fréquemment des consignes destinées à faciliter la navigation de l'internaute sur le site. Dans ce sens, les verbes métacommunicatifs, sous forme d'impératifs et d'infinitifs, y sont abondamment attestés pour indiquer les manipulations à effectuer lors de la lecture de la page: «Téléchargez » (UDC), « Continuer» (PS), « Rechercher » (AS)... De même, le teasing est couramment pratiqué: la page comporte des énoncés fragmentaires suivis de consignes allocutées ("Lire », Verts; "En savoir plus », PLS...) que l'internaute est invité à cliquer pour poursuivre sa lecture dans le corps du site.

D'un autre côté, le dialogisme interlocutif amorce la dynamique d'un échange entre les auteurs du site et ses visiteurs, cela aux différentes phases de la communication politique mise en place. Ainsi, le contact avec les internautes est activé par le recours aux formules de bienvenue (8 pages sur 16), celles-ci étant

5. Ces sites monolingues peuvent aussi attendre des internautes une capacité d'adaptation à leur langue.

6. Sur ce concept, voir S. Moirand, 2002, «Dialogisme », P. Charaudeau et D. Maingueneau éd., Dictionnaire d'analyse du discours, Paris, Le Seuil, p. 176. 
parfois motivées par le contexte temporel (souhait de bonne année 2005 sur la page des Grünliberale) ou connotées idéologiquement: «Socialement vôtre » (PS). Les visiteurs se voient en outre impliqués dans le discours politique luimême, grâce à des questions ouvertes simulant l'ébauche de débats («Fautil privilégier la culture d'État?», PRD...). Ils sont enfin sollicités pour effectuer des actions politiques ciblées, stratégie énonciative largement pratiquée par l'Union démocratique du Centre («Signez maintenant», «Soutenez le Comité d'action »...).

Ces traits dialogiques sont très variables selon les partis, indépendamment de leurs tendances politiques. Ils sont absents de seulement deux pages d'accueil (GB, PCS), ce qui oriente leur site vers un monologisme prédominant et une posture autocentrée. Dans la majorité des pages, on observe un dialogisme sélectif, axé sur les procédures informatiques à suivre (cas du PST) ou sur les rapports interpersonnels avec l'internaute (PES: «Vous nous donnez l'occasion de mieux vous connaitre »). Trois partis exploitent toute la palette des possibilités dialogiques: I'UDC, le PDC et les Verts. La page d'accueil de ces derniers accorde presque autant d'importance à la relation interlocutive qu'au message communiqué, en associant le dialogisme exploratoire du site («Prenez quelques instants et visitez notre site »), le dialogisme de politesse (salutations renouvelées) et le dialogisme spéculatif (nombreuses incitations à l'échange d'idées). Ces traits allocentrés réitérés contribuent à la posture de convivialité et d'ouverture que veulent se donner les Verts.

\section{Des iconotextes hybrides}

Les pages d'accueil des partis politiques suisses se remarquent par leur hybridation, qui résulte de leur structure concise. Outre qu'il s'agit d'iconotextes ${ }^{7}$ combinant les codes de l'image et de l'écriture, elles oscillent entre les genres incitatif et informatif, tout en concentrant une pluralité de fonctions.

\section{Mises en texte modulables}

La textualisation des pages d'accueil des partis politiques suisses s'avère inégale et ambivalente. Appartenant au genre incitatif et répondant à une stratégie promotionnelle, les slogans constituent un élément identitaire présent dans huit cas sur seize. Placés en haut de la page à côté du nom des partis dont ils fournissent la devise, ils ont une forte portée mobilisatrice, selon plusieurs orientations: universalisantes (PDC: «Le parti suisse!»), programmatiques

7. Au sens de M. Nerlich, 1990, «Qu'est-ce qu'un iconotexte?», A. Montandon éd., Iconotextes, Paris, Ophrys, p. 255-302. 
(PLS: «Pour une Suisse qui gagne »), prospectives (GLP: «Jetzt. Aber auch morgen $»^{8}$ ), interlocutives (Lega: «Diamo Forza al Ticino! » $\left.{ }^{9}\right)$...

La barre-sommaire, le titre et le chapeau-résumé se rangent quant à eux plutôt dans le genre informatif. Tandis que les deux premiers éléments sont toujours attestés, le chapeau apparait facultatif (9 fois sur 16). Pour leur part, les titres jouent un rôle de synthèse de l'information, mais aussi d'accroche du visiteur et de cohérence structurale de la page (agencement fréquent selon un ordre chronologique décroissant: GB, PLS, PS, Verts...). En fait, le grand intérêt de ces trois éléments est qu'ils révèlent deux conceptions informatives opposées de la page d'accueil, quelle que soit l'idéologie des partis. Mettant l'accent sur le cadrage cognitif, plusieurs pages d'accueil optent pour une conception thématique de l'information, se limitant à la barre-sommaire et à des titres-rubriques. Souvent minimaliste, la mise en texte s'articule à ce moment sur le connu ou le reconnu, comme le montre la page de l'Union démocratique fédérale. Sa barre-sommaire comporte un étiquetage des rubriques classiques qu'on peut attendre d'un parti politique: «Présentation du parti » - «Élections » - «Programmes d'action », etc. Et son rédactionnel se borne à quatre titres isolés qui balisent des évènements politiques dont les citoyens suisses sont parfaitement au courant: « Référendum contre le Pacs fédéral » - «Référendum contre la loi sur la transplantation »... On peut parler ici de page d'accueil au sens étroit du terme, qui propose une sélection et une annonce d'informations dont l'internaute découvre le traitement dans le corps du site par cliquage sur les liens prévus.

À l'inverse, d'autres pages débordent l'accueil pour effectuer un traitement résolument rhématique ${ }^{10}$ de l'information, privilégiant l'apport d'informations nouvelles qui demandent à être déjà lues en elles-mêmes, sans qu'il soit nécessaire de visiter le reste du site. Ces pages mettent dès lors en avant le couple titre-rhème - chapeau qui revêt plusieurs configurations. Tantôt ce couple se déroule sur la page sous forme de listing de blocs informatifs donnant le point de vue du parti sur différentes composantes de l'actualité (PLS). Tantôt l'apport informatif se fait par le biais d'une mise en gros plan. Ainsi procède le Parti socialiste lorsque, sous la rubrique "Focus », il commente deux évènements marquants du moment: le raz-de-marée en Thaïlande et les accords bilatéraux avec l'Union européenne. Tantôt le développement rhématique de l'information est traité en très gros plan, comme sur la page du Parti démocrate-chrétien. Celle-ci contient un seul titre orienté argumentativement («Rouges-Verts: politique à hauts risques »), lui-même suivi non plus d'un simple chapeau, mais d'un véritable article de 36 lignes de son secrétaire général, Reto Von Nause,

8. «Maintenant. Mais aussi demain. »

9. «Donnons de la force au Tessin!»

10. Pour la distinction thème-rhème, voir B. Combettes, 1983, Pour une grammaire textuelle, Paris, Gembloux, De Boeck-Duculot, p. 29 et suiv. 
contre la politique à ses yeux laxiste de la majorité Rouges-Verts du législatif de la ville de Berne.

Si, malgré leur caractère composite, la plupart des pages d'accueil parviennent à une certaine cohérence textuelle, on relève un cas équivoque: celui de l'Alliance socialiste (reproduction en Annexe 2). Ce site hésite en effet entre deux structures distinctes, sans vraiment les concilier: celle d'une page d'accueil pour un parti politique (avec la barre-sommaire relative à ses activités: "Agenda », "Communiqués »...) et celle de la une pour le journal Solidarités de ce parti (éditorial, ours, etc.). Un tel amalgame non résolu rend difficiles la compréhension et la lecture de cette page d'accueil, ne serait-ce que par la multiplication des éléments rédactionnels qu'il occasionne.

\section{Organisation scripto-iconique}

La dimension textuelle des pages d'accueil des partis politiques suisses est toutefois indissociable de leur composante iconique, l'ensemble faisant de celles-ci des unités sémiotiques ou des iconotextes qui reflètent l'identité des partis concernés. Par-delà le particularisme de ces pages, deux grands types de configuration prédominent.

Au niveau local, le code scriptural et le code iconique tendent à fonctionner en complémentarité. Cela se vérifie avec le point focal qu'est le logo, présent en attaque du parcours de lecture sur toutes les pages visitées. Opérant comme signature, le logo permet la personnalisation des partis et, par son caractère en principe connu, leur identification sans peine de la part des internautes. Exceptionnellement, il prend la forme d'un seul dessin, revêtant alors une nette fonction symbolique. Ainsi, l'internaute helvétique est enclin à adhérer à la valorisation patriotique et sentimentale du logo des Démocrates suisses qui insère la croix blanche du drapeau national dans un cœur rouge. Le logo peut être alphanumérique, à l'instar de celui de l'Union démocratique fédérale, avec son sigle composé en capitales italiques. Mais l'écriture en violet du nom de ce parti lui assure une iconisation minimale, laquelle accroit son impact visuel. La plupart des logos sont en fait scripto-iconiques, condensant les fonctions identitaire et symbolique. C'est le cas de celui du Parti écologique qui réunit une fleur et une feuille stylisées, elles-mêmes suivies du nom du parti écrit en vert. Par ailleurs, si la majorité des pages d'accueil (11 sur 16) contiennent des images sous forme de vignettes, ces dernières n'acquièrent leur sens qu'à travers la médiation des énoncés qui leur sont associés, ce que montre la page d'accueil du Parti socialiste. Soit ses vignettes ont une fonction illustrative quand elles confirment iconiquement l'information formulée dans le textuel (reproduction d'un village ravagé en face du titre: «Le PS solidaire des victimes du raz-de-marée en Asie »), soit elles ont une fonction allégorique quand elles emblématisent l'information du titre (représentation du drapeau européen intégrant une Suisse rose - couleur 
du Parti socialiste - à côté du titre: « Notre refus d'une Suisse qui s'enfermerait dans l'isolement »). Plus rarement, les vignettes ont une fonction locutive par rapport au texte, lorsqu'elles figurent le portrait de l'auteur d'une citation mise en exergue (cas de la page d'accueil du Parti écologique représentant Ueli Leuenberger, son vice-président).

Au niveau global, on observe une iconisation d'ensemble des pages d'accueil par la couleur qui relève du code plastique. La colorisation qui en résulte répond à deux objectifs. En premier lieu, elle amplifie sur la page l'identité visuelle des partis, surtout lorsqu'elle diffuse la couleur de leur logo. Entre autres, le bichromatisme orange-bleu du logo du Parti démocrate-chrétien est repris par la suite comme fond (orange) des rubriques des deux barres-sommaires, ou comme couleur (bleue) de leurs sous-titres. En second lieu, la colorisation consolide la cohésion du parcours de lecture des pages d'accueil, comme celle du Parti libéral, dont la lisibilité des titres est rythmée par la réitération du caractère bleu ciel utilisé. La colorisation compense à l'occasion le manque d'images. Ainsi, la colorisation en bleu (pour les fonds des rubriques) et en violet (pour les titres) de la page d'accueil du Parti chrétien social atténue sa dimension essentiellement langagière. La colorisation sature parfois la lecture de la page, à l'exemple de celle du Parti suisse du Travail dont le chromatisme rouge - indice d'un ancrage à gauche - systématiquement employé pour le fond des rubriques de la barre-sommaire et pour les titres du rédactionnel, finit par introduire une indifférenciation qui peut désorienter le visiteur. Dans tous les cas, le rôle majeur accordé à la colorisation assure la prédominance de la visibilité sur la lisibilité. Une visibilité qui renforce la mise en spectacle du politique sur les pages en jeu, chaque fois singularisées en des structures figuratives hautement perceptibles, dont la force de contact ${ }^{11}$ garantit une reconnaissance et une mémorisation rapides.

\section{Un positionnement communicatif flou}

Par-delà l'hybridation de leurs structures, les pages d'accueil des partis politiques suisses posent le problème de leur finalité communicative, laquelle s'articule autour de la notion de présentation. S'agit-il de présenter un site informatique, de se présenter ou de présenter des évènements liés à la vie politique? Alors que le premier objectif est plus ou moins satisfait par la navigabilité des pages d'accueil examinées, les deux autres donnent lieu à un traitement ambigu.

11. Pour des compléments sur l'attractivité visuelle de la page d'accueil, voir G. Gulati, 2004, « Members of Congress and presentation of self on the World Wide Web », The Harvard International Journal of Press/Politics, ${ }^{\circ}$ 9-1, p. 22-40. 


\section{Autoportraits déficitaires}

Selon les recommandations de Franck Stauss ${ }^{12}$, la page d'accueil d'un parti politique devrait déjà nous en fournir une première description. Or, si les partis politiques suisses soignent leur identité visuelle, leur signalétique est ordinairement lacunaire au niveau des pages d'accueil, à part leur adresse régulièrement indiquée. Ainsi, celles-ci renferment très peu d'éléments sur la vie interne des partis. Seul le Parti radical démocratique fait une courte allusion au renouvellement de son appareil, et l'Alliance socialiste consacre une nécrologie à l'une de ses dirigeantes. De même, les modalités d'adhésion ne sont pratiquement jamais évoquées dans le rédactionnel, excepté sur la page de l'Union démocratique du Centre: «Êtes-vous déjà membre? " De plus, très peu de partis font explicitement état de leur idéologie et, lorsqu'il en est question, cela s'effectue rapidement à travers un bref slogan qui les situe sur l'échiquier politique: « Parti libéral. Le parti d'une droite efficace et moderne. »Quant aux programmes politiques, ils sont constamment passés sous silence, à part sur la page du Parti écologique qui expose clairement sa vision: « Nous nous engageons pour la protection de l'environnement, pour le maintien des ressources naturelles et pour la justice sociale, économique et politique [...]».

De toute évidence, le propos des pages d'accueil analysées n'est pas de brosser un portrait - même élémentaire - des partis suisses, portrait qui est en fait rejeté dans les niveaux inférieurs de plusieurs sites. Cette déficience en autodescription institutionnelle tient au public visé. Pour les adhérents ou les sympathisants, une telle autodescription est superflue, dans la mesure où elle fait partie de leurs présupposés. Aux yeux des internautes curieux, une présentation purement descriptive des partis manquerait d'attrait et une sollicitation trop expresse à en devenir membre pourrait être perçue comme une agression territoriale, ayant un effet de repoussoir. Mais si, paradoxalement, les partis politiques suisses se présentent peu dès leurs pages d'accueil, c'est surtout parce qu'elles sont davantage une vitrine publicitaire qu'une source de renseignements. Dans cette optique, la monstration du faire des partis face aux évènements leur assure un impact autrement plus stimulant que la déclinaison de leurêtre.

\section{Ciblage du discours sur l'actualité immédiate}

La quasi-totalité des pages d'accueil visitées mettent en scène l'engagement des partis politiques dans les évènements du moment: le tsunami en Asie du

12. F. Stauss, 2004, «Kampagne: Politik als Marke. Frau Kurasten sucht Orientierung », Politik \& Kommunikation, $\mathrm{n}^{\circ} 15$, p. 24-26. 
Sud-Est, les négociations bilatérales avec l'Union européenne, le forum économique mondial de Davos... Cela est confirmé par le renouvellement rapide du contenu de ces pages ${ }^{13}$, par la datation courante et méthodique de leurs rubriques (12 cas sur 16) et par la réitération de mots-vedettes appartenant au champ sémantique de l'actualité: « actuel », «news »... (13 pages sur 16). Suggérant indirectement le positionnement idéologique des partis, cet ancrage engagé sur le contexte immédiat apparait à travers trois grands procédés langagiers. D'un côté, on relève de nombreux énoncés performatifs qui manifestent un discours d'action en prise sur les faits et les problèmes. Énonciatèmes de refus: « Non à l'extension de la libre circulation des personnes » (UDC); actes de langage promissifs: "Le PCS suisse garantit un pacte social équitable », directifs: «Le comité libéral exige un débat soutenu » ou désapprobateurs: «Le PST/ POP s'oppose à l'attaque contre les droits à une retraite digne »... D'un autre côté, cette tendance à la performativité se double d'une énonciation axiologique, avec des évaluatifs à la hausse ou à la baisse: «Blocage inadmissible des centres de tri de la Poste » (PLS). À cela s'ajoute un penchant pour la dramatisation, avec un appel aux émotions: «La tragédie de l’Asie du Sud restera gravée dans les mémoires. Les victimes principales en sont une fois de plus les pauvres et les enfants » (PST). Tous ces procédés répondent à une stratégie de sensibilisation empathique de l'internaute, cependant qu'ils établissent une proximité affective et intellectuelle avec lui, interpellé par des sujets qui le touchent peu ou prou.

De tels procédés sont plus ou moins attestés selon les pages d'accueil, se traduisant par différentes textualisations. Assez rarement, on remarque des pages-communiqués succinctes, comme celle du PRD, qui estompent énonciativement leur implication dans l'actualité. Plus souvent, et surtout dans les sites de gauche, on a affaire à des pages-mots d'ordre affichant des déclarations de principe et un positionnement péremptoire: «Zum Anti-WEF Protest vom 22. Januar 2005. Eine andere Welt ist nötig! $!^{14} »(\mathrm{~GB})$... Cinq autres pages d'accueil, dont deux de partis centristes (PDC, PLS) et trois de formations de gauche (Verts, PS, PST), constituent des pages-tribunes, avec un positionnement militant circonstancié et étayé argumentativement. Ainsi, chacun des sept blocs rédactionnels de la page du Parti socialiste exploite amplement la gamme de l'argumentation rhétorique. Entre autres, le long chapeau consacré aux accords avec l'Union européenne pour la libre circulation des personnes entremêle les procédures du pathos («Le PS est heureux de constater que [...] le

13. Nous avons constaté ce renouvellement par nos vérifications ultérieures du 17 février et du 9 mars 2005. À part un ou deux partis marginaux - telle la SGA - qui se réactualisent peu, ce renouvellement est soit total (la page de l'Alliance socialiste change entièrement tous les dix jours), soit partiel. Ainsi, même si la teneur de sa rubrique "Communiqués » a été modifiée, la page du Parti socialiste présentait toujours, le 17 février 2005 , le « focus » du 13 janvier.

14. «Pour la manifestation anti-WEF du 22 janvier 2005. Un autre monde est nécessaire ! » 
syndicat UNIA s'exprime en faveur d'une ouverture de la Suisse à l'Europe ») et la rigueur du raisonnement logique:

Cette ouverture est dans l'intérêt [...] de la place économique suisse. Un rejet de ce nouvel accord bilatéral aurait non seulement de graves conséquences sur tout le processus de rapprochement de notre pays avec l'Union européenne, mais il interdirait aussi l'entrée en vigueur de mesures de protection des personnes salariées durement acquises.

Ce faisant, le Parti socialiste se construit un ethos favorable, fait de responsabilité et d'ouverture. De surcroit, cette implication dans le débat politique conforte la fidélisation des militants et, par sa portée positivante, elle peut transformer - dès la page d'accueil - les visiteurs étrangers au Parti socialiste, mais pro-européens, en sympathisants de ce dernier ${ }^{15}$.

\section{Quelle représentation du politique?}

Finalement, les pages d'accueil des partis politiques suisses se caractérisent par deux dominantes:

- elles mettent en œuvre une stratégie de l’immédiat, liée au support Internet. Elles doivent en effet, dès leur apparition sur l'écran, accrocher l'intérêt de l'internaute par l'accessibilité de leurs liens, par leur visibilité, par leur lisibilité et par leur implication de celui-ci, tant communicative (dialogisme) qu'informative (mise en scène engageante de l'actualité). En somme, elles ont d'abord une fonction phatique ${ }^{16}$, fondée sur l'établissement et la conservation du contact avec le visiteur;

- ces pages d'accueil offrent une vision brouillée du message politique en raison de leur discours promotionnel prépondérant, au détriment d'un discours véritablement citoyen. Quelle que soit au demeurant la couleur politique des partis suisses, leur objectif est avant tout de montrer une image attractive et dynamique d'eux-mêmes qui les distingue ${ }^{17}$ des partis concurrents, indépendamment des sujets abordés. Cette propension à l'autopromotion est perceptible avec la mise en relief proprement mercatique de l'identité visuelle des partis (par le biais de logos et d'une colorisation systématique), avec leur emploi fréquent d'un langage incitatif et rhématique, ainsi qu'avec leur recours au merchandising ${ }^{18}$.

15. Suivant en cela la procédure décrite par H. Yildiz, 2003, «Internet : un nouvel outil de communication multidimensionnelle », V. Serfaty éd., L'Internet en politique, op. cit., p. 285-286.

16. Selon la terminologie de R.Jakobson, 1963, Essais de linguistique générale, Paris, Minuit, p. 217.

17. Dans l'acception de P. Bourdieu, 1979, La Distinction, Paris, Minuit, p. 26-27.

18. Entre autres, les pages d'accueil des Verts et de l'UDC comportent respectivement une rubrique "Commande » et «Shopping UDC ». Si l'on ouvre cette dernière, on nous propose de commander des affiches électorales, mais aussi divers articles au sigle de l'UDC (couteaux d'officier, cartes à jouer, briquets, montres, polos...). 
Une telle propension à l'autopromotion soulève en outre deux problèmes. D’une part, celui de la dénomination même de pages d'accueil pour la majorité des occurrences que nous avons analysées. Certes, comme on l'a vu, cellesci accueillent le visiteur en lui présentant (généralement assez peu) des partis et en le guidant dans la découverte de leurs sites. Mais c'est pour tout de suite réorienter leur discours vers une captation du public dans l'univers praxéologique et idéologique qu'elles mettent en spectacle. Captation que les internautes sont toutefois libres d'accepter ou de refuser. D'autre part et plus largement, se pose la question du bien-fondé de l'appellation - évoquée en introduction de démocratie électronique utilisée pour qualifier ce type de pratique politique. En effet, ces pages d'accueil érigent l'image d'un internaute davantage consommateur de politique qu'agent du débat politique. En cela, même si les rubriques sous-jacentes des sites en question et la multiplication de ces sites sur Internet peuvent introduire des formes de débat, leurs pages d'accueil témoignent de l'importance des contraintes médiatico-publicitaires qui uniformisent et standardisent peu à peu la communication politique.

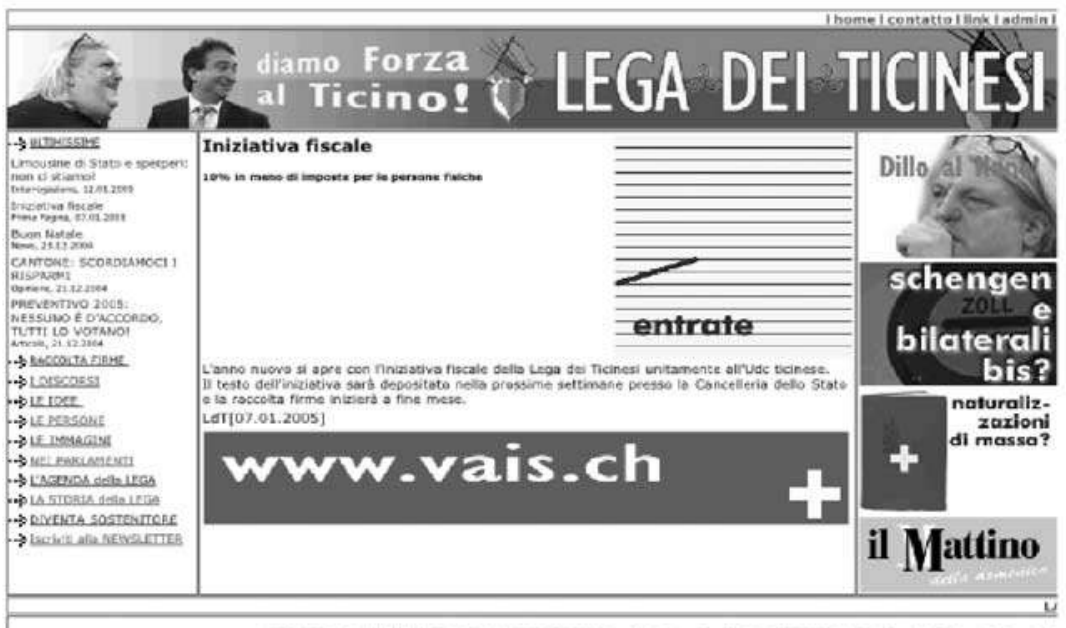

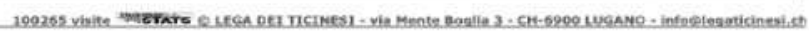

Annexe 1: page d'accueil de la Lega dei Ticinesi 


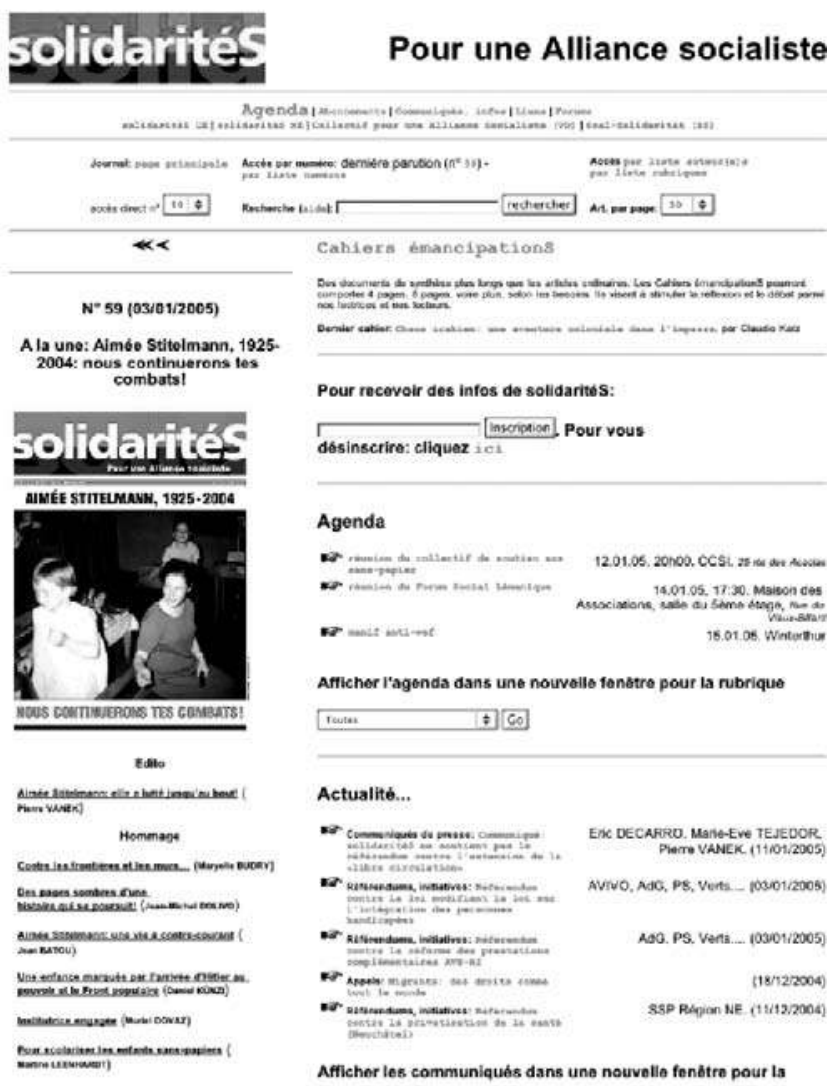

Annexe 2: page d'accueil de l'Alliance socialiste 